\title{
CHAPTER SIX
}

\section{Source Theory and the Philosophy of Law}

\section{The law as a subsystem}

The law is a compartmentalized subsystem within every developed culture. In most (if not all) of those cultures we can find a definite, separate set of data that are known as laws. These data are transmitted by unique sources - the legislature, the courts and the like - that are not identical to those of the larger system, though not completely detached from them. These sources not only transmit the data, but also determine their being laws (the rules of recognition, in H. L. A. Hart's terms). In jurisprudence they have for ages been termed "sources of law", a terminology that anticipated that of Source Theory, and so it almost invites an analysis in terms of Source Theory.

Indeed, problems in the philosophy of law too can be expressed, and perhaps even solved, in terms of Source Theory. Since I cannot discuss all possible legal systems here, I will choose for my test case the modern legal system - the rational, Western one, a subsystem of WRS - which most of philosophers of law have considered. Here, too, I will not discuss a specific legal system but will take an ideal type as an object for analysis. This can be called the Western Rational Legal Subsystem. Nevertheless, at least some of the arguments I present here are true, mutatis mutandis, of more traditional legal systems as well.

Even though in every culture the law is a subsystem of the general cultural system, it does not always have the same degree of attachment to the parent system. Max Weber (1978, Volume II: 656) distinguished between formal (or formalistic) and non-formal systems. He defined a formal system as one that does not decide the law "upon an ethical, emotional or political basis" (ibid). To formulate Weber's stand more sharply and more radically (even if Weber himself would probably disapprove of this alteration), we could say that the Weberian ideal is that of an autarchic subsystem detached from the parent

How to cite this book chapter:

Brown, B 2017 Thoughts and Ways of Thinking: Source Theory and Its Applications.

Pp. 103-117. London: Ubiquity Press. DOI: https://doi.org/10.5334/bbh.g. License: CC-BY 4.0 
system, which is supposed to provide data from within itself. At first it would seem that it is supposed to provide an answer for every legal question without using any additional sources; and, indeed, Weber defines the formal system as "gapless" (ibid.: 657-658); However, as we shall see, such complete detachment is impossible, and the most that one can consider is the maximal degree of detachment.

We begin the discussion with the fundamental question of all political or legal philosophy, the question of authority. Afterwards we will discuss the sources of judgment and the application of judgment to facts.

\section{Authority and its justification}

The problem of authority is one of the oldest and most important in political philosophy and the philosophy of law. For the purposes of the present discussion we will define authority as one person's right to obligate another individual to obey his orders. This person could be a king, a legislator or any other person who claims to have legal authority. (Actually this applies, mutatis mutandis, to ethics as well, even though it was not legislated by any particular person, but for this reason we will leave it out of the discussion, to avoid complications). The question it raises is thus what gives the former person this right. The answer to this question, insofar as the responder affirms that this person actually has such authority, is that he has gained it by virtue of principle p. p is a sentence, and therefore it is transmitted by a source - let's call it $\mathrm{d}$. The real authority behind $\mathrm{p}$ is therefore $\mathrm{d}$. If $\mathrm{d}$ is a person, we can ask further who or what gives him/her that authority, and so on. In terms of Source Theory, a person with authority is nothing but a source (of orders), while the people who obey him have adopted this source (whether by choice or coercion). Thus the problem of justifying authority is parallel to the problem of justifying adoption in Source Theory. Both involve an infinite regress in a peculiar version stemming from the source-datum relation (See Raz 1970: 102). The principle p can therefore be considered the authority justification principle. (In philosophical discourse, the term "legitimation" is generally used to denote the justification of authority, so I will use it occasionally in place of "justification").

The legal philosophy that seems most suitable to Source Theory is Hans Kelsen's Pure Theory of Law (Kelsen 1965; see also Kelsen 1961, Chapter 10). Kelsen described law as a hierarchic structure of norms often equated to a pyramid. In this structure, most of the norms attain their validity from "higher" norms. Thus, municipal enactments attain their validity from the law of the state and the law of the state attains its validity from the constitution. At the top of every such system there is a basic norm of "You must obey x" that gives validity to the entire system. In a monarchy, for example, $\mathrm{x}$ is the king, but if some rebel topples that king, that basic norm will soon be changed. The 
basic norm itself is not justified by anything further; otherwise it would not be basic. As the ultimate justification for all the norms in the system, the basic norm cannot itself be subject to justification. It has been rightfully contended that Kelsen's theory is one of legal-theoretic foundationalism. It has received numerous critiques, many of them attacking it exactly for its foundationalist character. Source Theory can provide some defense for Kelsen's theory, but not in its entirety, as will be discussed below. The basic norm can be described as none but an adoption sentence, and the $\mathrm{x}$ of the basic norm is none but a superior source in the source model of the legal system. There is, however, one important distinction: in Kelsen's theory, law is a system, while in Source Theory, law is a subsystem. This is not just a matter of labeling. Kelsen is completely indifferent to the cultural environment in which law is established and of which law is a part. Being a staunch positivist, he is eager to detach law from morality, viewing the basic norm as not a moral norm but a "pure" legal one. He therefore also detaches law from the normative conventions of the society in which law works. These norms, however, are those that give the basic norm, and therefore the whole legal system, its legitimacy. Legitimacy is the sociopolitical twin of the epistemological "justification". As a result, these legitimizing norms prove superior to the basic legal norm. This point will be addressed further below in this chapter.

But the applicability of Source Theory to law does not necessitate a full commitment to the Pure Theory of Law over other theories. Kelsen's idea of a basic norm is not very far from H. L. A. Hart's "rule of recognition" (Hart 1961, Chapter 6; see the analysis in Marmor 2011, Chapters 1-3). Although Hart himself alluded to differences between the two concepts (Hart 1961: 105), it is quite clear that both of the rules serve as foundational elements of the legal system and can be considered adoption sentences. The claim of Bentham $(1945,1960)$ and Austin (1995) that the sovereign is that foundational element can also be reformulated in the terms of Source Theory, i.e. that the sovereign is a superior source in the model. Raz (1970), who coined the term "chain of validity" to describe the order of authorization in a way quite similar to our "chain of adoptions", claims that usually legal systems are defined by more than one source of authority (see Raz 1970, 1986, 1990), and so his theory calls for its reformulation in terms of "source model". In light of the insight I presented in Chapter Three, which claims that the issue of adopting a source or a system is essentially normative, we can say that the issue of the justification (legitimation) of authority is almost fully parallel to the issue of the justification of adoption.

In modern political philosophy, several principles have been suggested for justifying authority. Each of theories that have been presented in the history of political philosophy, such as those that determine the justification principles by "natural law", "social rules", "the social contract", or "the divine right of kings", assumes that its particular principle is self-evident. There is, however, reason to ask what the source of this principle is and what justification there is for its 
authority. To be precise, the source of this justification is not merely a truth source, but also an authority source, since it demands obedience, and, in this case, obedience to its command to grant authority to the authoritative body it "chose" by virtue of the principle it transmitted. As in epistemology, there is no escape here from the claim that the last justification is always internal - that is, arbitrary.

An especially interesting theory on this issue is offered by Joseph de Maistre, according to Owen Bradley's interpretation (1999, Chapter 5; its grains can be found in Huber 1958 and Lebrun 1965). Although this interpretation does not seem to be perfectly faithful to the French philosopher's thought, it is too brilliant and challenging to be abandoned; for precision's sake we will therefore call it the "Maistre-Bradley theory". To sum up the theory simply and briefly, it claims that all authority was originally acquired by force - that is, by violence (a similar theory was previously suggest by Paine 1792, who used it to mock the traditional authority, and later by Freud,1933). When this authority is maintained over time, it acquires legitimacy, which makes violence unnecessary. On the contrary, this legitimation is intended to deprive others of the right to use violence so as to obtain the authority. For this authority to become established, it must hide its historical roots. Therefore real authority can never be based on a particular event or document, but rather on a hidden, unrecognizable source. This provides the authority with a mysterious aura of "holiness" or "divinity", which makes it self-evident and allows it to be accepted. To quote Bradley:

[L] egitimacy does not derive from "birth" but from simple longevity: "Everyone knows that there are fortunate revolutions and usurpations very criminal in their beginnings, to which, however, it pleases Providence to affix the seal of legitimacy by a long position". Maistre indeed expresses the suspicion that no great power has origins as "humble" as they might appear: "At the present day, with our philosophy, all our civilization, all our fine books, there is not, perhaps, one European power in a position to justify all its possessions in the face of God and Reason"...

Maistre presents this same general claim in an even more provocative form. In the early history of constitutions, "everything reduces to what I have called legitimate usurpation; the sovereign acts, obedience is general, tranquil, and constant; the option, if there is one, is particular, turbulent and temporary; finally, the sovereign sits down, and on his throne is written: I possess because I possess". This is legitimacy!

For Maistre the beginnings of sovereignty, its "birth", if not its acquired name, are always illegitimate: hybrid, puny, inglorious, vague, and bloody (Bradley 1999: 112-113).

Despite this theory's cynical charm, however, it does not provide a solution for the essential problem. In effect, it gives up on the possibility of justifying 
authority. If violence is the justifying principle, then we can ask what justifies this very principle. One may suggest that the justifying source is some "unknown source", but this does not advance us any further. Its invisibility may make it an effective practical instrument on the level of the Machiavellian enterprise (which the Maistre-Bradley theory discusses), but it does not provide any answer on the level of philosophical justification. On this level, an unidentified source not only fails to serve as a good justification for an argument, but may even weaken it.

We therefore come to the same conclusion here about the concept of authority as we did in the epistemic arena about the concept of truth. This concept is meaningful only within a particular system whose basic sources are the ones that transmit the justification principle. The basic sources themselves do not need to be justified, since their justification is necessarily internal, that is, arbitrary. This characterization, which is pertinent for logical systems, is also correct, mutatis mutandis, for the social arena, where a cultural system is involved, and consequently for its normative subsystems. All of the "truths" in the system are those that it transmits and endorses, including normative truths. One of these truths is authority. This is the root of the centralized authority of the state (which is sometimes called "sovereignty"), including the authority of its various powers, one of which is the law. Since the law is also a system, it should be seen as a subsystem of the cultural one (see Mautner 2011, and the different approaches and rich references presented there).

Since the law's authority is derived from that of the cultural system, a situation in which the legislature enacts norms that are at odds with values accepted in the cultural system is quite problematic. Such a situation can be defined as a contradiction between two norms within the same cultural system: a substantive norm, which in this case is a value accepted by the cultural system; and an "empowering" norm, which is the one that authorizes the legislature to enact laws. As in cases where there are contradictions among the data in the epistemic arena, the source model is called upon to solve the problem through the division of labor, particularly through the hierarchy. The nature of the hierarchy that can accomplish this is the subject of the controversy in the philosophy of law between the naturalists (who advocate the theory of natural law) and the positivists. The former place the substantive norms higher on the hierarchy, with the empowering norms subordinate to them, while the latter advocate the opposite order.

\section{How laws are made}

The legal process is one in which the law is applied to the facts. There are thus two levels of discussion, that of the law and that of fact. The faculty of judgment is apparently the sole factor in this process, since the legal process 
connects general norms to individual cases through modus ponens: All those who steal things are liable to three years' imprisonment; John stole something; therefore John is liable to three years' imprisonment. This is the traditional conception of the legal process. Although Hage (1996) suggested a different calculus, based on a nonmonotonic logic, which claims that judgment is defeasible, for the purposes of our discussion I will ignore this novel idea and merely note that the essence of what I have to say here can be applied, mutatis mutandis, to this new logical conception of the legal process as well. I will therefore base my discussion on the conception of the legal process as the application of modus ponens. However, we must first understand how this is done, by analyzing the way the courts determine each of the levels, that of the law and that of the facts.

Judgments are determined by their sources. As I mentioned above, the term "source", which we have been using throughout all our discussions in this book, was developed mainly for the theory of law, and the term "sources of the law" was probably used in jurisprudence much before the term "sources of knowledge" was commonly accepted in epistemology. The classic theory of law recognizes various sources of law: written law, which is enacted by a legislative body; precedent, which is determined by the courts; custom, which is generally decided by the community, or at least by parts of it; and the like. The two great traditions of Western culture - the Continental and the Anglo-American have been divided on the issue of the hierarchy of these sources. However, in most modern law systems the written law has the highest status in the hierarchy, while precedent and custom are valid only through its force. In any case, this will be the starting point of our discussion here, if only for the purpose of simplification.

Since the law is a general norm, from the standpoint of Source Theory it is a general datum. Underlying the laws are values, or principles, which the legislators seek to protect. In the simplest cases, each law embodies one value, but cases of this sort are actually very rare. In general each law embodies a number of values, as well as the balance among them as determined by the legislature. Since a logical analysis of the layers of such laws would be very complex, we will consider a very simple example here. With such an example, where the legislators are defending only one value, the defense is categorical. Thus the defense of this value takes the form, "Any action that is contrary to value $\mathrm{v}$ is prohibited", or, in more extreme and very rare cases, "Any action that promotes the value $\mathrm{v}$ is obligatory". Nowadays legislators generally do not express their orders in terms of prohibitions or obligations, but rather in terms of the sanctions to be imposed on anyone who acts in contradiction with the stated order. This is quite clear in criminal law, but it is also true of civil law, in which the protected value is generally private property and the sanctions are generally of the civil sort. As a result, prohibitions are now expressed in the form, "Those who perform act w will be sanctioned in the following way", while obligations 
are expressed in the form, "Those who do not perform act w will be sanctioned in the following way". However, this change of form should not divert us from the true normative content of the law, which is a prohibition or an obligation.

Consider, for example, the law that prohibits discrimination in hiring. The value that the law is protecting is equality. We can therefore analyze the layers of the law as an argument with this structure:

Acts that are opposed to the value of equality are prohibited.

Discrimination in hiring is an act opposed to the value of equality.

Conclusion: Discrimination in hiring is prohibited.

Let us analyze this argument with the classical distinction between normative and descriptive statements (ignoring its philosophical difficulties for the moment). In this example, both the major premise and the conclusion are normative statements, while the minor premise is a descriptive one. This is the case because it states that the concept "discrimination in hiring" is included in the concept "an act opposed to the value of equality". Even though this statement refers to normative concepts, it expresses the relationship between the concepts, which is a descriptive one. We can therefore say that the analysis of the law has the following layers: a normative major premise, which determines the higher norm, or the value; a descriptive minor premise; and a normative conclusion. Thus the justification of the law requires the following sources: one that justifies the higher norm; one that justifies the description; and one that justifies the logical deduction, that is, the conclusion.

Now consider the following example:

Acts that are opposed to the value of not risking human life are prohibited.

Driving at more than $70 \mathrm{mph}$ is an act that is opposed the value of not risking human life.

Conclusion: Driving at more than $70 \mathrm{mph}$ is prohibited.

In this example, too, the major premise and the conclusion are normative statements, while the minor premise is a descriptive one. However, the minor premise is not transmitted by the analysis of concepts but rather by experience - that is, the senses, with the aid of abstraction, which tell us that driving at more than 70 mph risks human life.

In ordinary legal proceedings, the major premise is a general datum, i.e., a sentence with the universal quantifier, while the minor premise applies it to a particular person. For example:

All those who steal are liable to three years' imprisonment.

John stole something.

Conclusion: John is liable to three years' imprisonment. 
However, we can also imagine a case in which the minor premise is also general:

All those who steal are liable to three years' imprisonment.

All politicians steal.

All politicians are liable to three years' imprisonment.

In a case of this sort, the conclusion, too, is a general datum, and therefore a law. This allows it to appear as the major premise of yet another syllogism - for example:

All politicians are liable to three years' imprisonment.

John is a politician.

Conclusion: John is liable to three years' imprisonment.

However, we must now ask what status a general datum has when it becomes a law. The answer depends, as usual, on the source. If the sources of the law in the system decide that this datum is a law, then it has the status of a law; if not, it has the status of a fact, which must be proven by evidence for each case separately. Law courts also recognize another situation, in which the datum can be a presumption. A presumption of fact is a descriptive datum about which one of the sources of the law - generally the legislature - rules that it should be considered like a proven fact. However, most legal systems distinguish between a conclusive (or irrebuttable) presumption and a rebuttable one. If the presumption is conclusive, then it is just as valid as any other law, since the conclusions drawn from it are also conclusive. In contrast, if the presumption is rebuttable, then its main force is that it transfers the burden of proof to those who are trying to defeat it.

Consider another example:

All citizens who are responsible for their actions have legal capacity.

All citizens over the age of 18 are responsible for their actions.

Conclusion: All citizens over the age of 18 have legal capacity.

The minor (factual) premise is clearly arbitrary. We all know citizens under the age of 18 who are more responsible for their actions than some citizens over the age of 18 , and citizens over the age of 18 who are not particularly responsible for their actions. The presumption that the legislature asserts here is conclusive, and they do not even bother to formulate it as a presumption, but draw the conclusion directly and formulate it as a law.

We can therefore state in summary that almost all laws are constructed out of several hidden layers. The datum that appears as the law itself is the "lower" datum in the pyramid of the syllogism, called "the legal rule" in the theory of 
law. The higher data are the considerations that led the legislature to enact the law. These data generally consist of two layers: the major premise, which is normative, and the minor premise, which is factual. At the top of the pyramid are the principles, which are normative data out of which the entire legal system is built.

This brings us to the questions of where the supreme principles of the legal subsystem are drawn from, and what is the origin of the factual data that serve as premises for its "lower" norms, As for the supreme principles, two alternatives are possible: Either they are principles of the cultural system in which the legal subsystem operates, or they are not. If the first alternative is correct, then the principles of the legal subsystem are justified by the principles of the cultural system. If the second alternative is correct, then they have no external justification - in other words, they are arbitrary. However, since the legal subsystem is generally a social phenomenon, such a situation hardly ever occurs, and when it does, it is considered problematic. Thus the supreme principles of the legal subsystem are generally drawn from the values of the broader cultural system. If the cultural system is WRS or one of its branches, then these values are moral ones (I mean moral in the secular-humanistic sense of the word). If the cultural system is a religious one, then these values are religious as well. As a result, in normal situations, the legal subsystem is not and cannot be autarchic, in contrast to Max Weber's postulation.

This is also true for the factual premises upon which laws are based. In the vast majority of cases these factual premises are not determined by the judge as "trier of fact" through a proceeding subject to evidence law, but are accepted by the lawmakers (legislators, judges, commentators) as true on the basis of common conventions. These conventions are accepted not by virtue of the legal subsystem, which does not deal with things of this sort, but because they are data within the general cultural system. As in the case of the principles, this case demonstrates the legal subsystem's dependence on the cultural system to which it belongs. Moreover, even if the legal system can be autarchic in the realm of principles, although this is not a healthy situation, in the realm of factual premises such autarchy is simply impossible. We need to keep this in mind for the next section of this chapter.

From a broader perspective, it would seem that Source Theory leads to normative relativism. A norm that is accepted within a particular system is justified by the basic sources of that system, and no source of any other system can validate or invalidate it. According to these claims, we cannot denounce cannibalism or murder based on "family honor" in cultures where they are accepted.

To be sure, this conclusion is derived from Source Theory in a purely formalist line of argumentation, and can therefore be considered as a normative version of the nihilistic absurdities, which are also derived directly in a similar line of argumentation. The way out of this relativism is likewise similar to the one we used in the case of the nihilistic absurdities - namely, the transition from a for- 
malist to a pragmatist line of argumentation. At first glance it would seem that this does not solve our current problem, since even in the sociological arena, there are ancient cultural systems in which cannibalism and "family honor" murders are valid norms. Indeed, we cannot invalidate the internal justification of these norms within their systems. However, just as we used the conservative rule for individuals in our discussion of the absurdities, we can use it here for the society as a whole. The conclusion is not that we should rid ourselves of any basic sources we have because they are particularistic, since such a move would make it impossible for individuals to think at all. Instead, the legal subsystem should stick to its "native" sources, the ones that are at the basis of its cultural system. This system is the one in which it developed historically and in which it can be justified. In contrast to the individual, who may sometimes take a stand of a non liquet (and even that is not so appropriate in the normative realm), the society cannot afford a condition of a normative vacuum or undecidedness. Therefore the state, which is the body within which the legal subsystem exists, is not value-neutral, and it is pointless for it to aspire to be so. On the contrary, a state adopts the sources of a particular cultural system with all of its values, and one of its roles is to embody these values in its laws. Consequently, it should not allow other cultural systems that exist within it the freedom to act as they please, unless their values are not opposed to the fundamental values of the state. This does not mean that it should prevent any other system from existing, but it can, and perhaps even should, prevent an alien system that exists within its borders from damaging its most fundamental values. This means that it the right of a legal system which embraces cannibalism to suppress a legal system that opposes it, if the latter exists within the political borders of the former, and that the people who do not embrace cannibalism should subordinate their norms to it. It is the right of the people who adopt the humanist legal system, which opposes cannibalism, to try to escape the one which embraces it, or even oppose it within the constraints of that system, but as long as they live within the state (or culture) which embraces it, they should accept its authority.

\section{How judgments are made}

Our discussion of the structure of legal norms also has some ramifications for the distinction between formalistic and non-formalistic (or value-oriented) legal systems (I am now using the term "formalism" in a legal-theoretic sense, in contrast to the way I used it above, which was in contrast to pragmatism). This is not a dichotomy but rather a continuum that can be formulated as follows: The more the legislators try to formulate the rules in more general terms, the less formalistic they are, and vice versa (Brown 2008).

It has been claimed that, in general, traditional normative systems tend to be more formalistic, while modern legal systems tend to be less so (Wigmore 
1981, Volume IX, §2405; Barak 2005, 30). To paraphrase Hobsbawm (1989: 4), the increase of non-formalistic interpretations of traditional formalistic norms demonstrates that they have become weaker. This is true only for traditional systems because formalism is one of the typical features of such systems, at least in their external appearance.

Law theorists have pointed out that the justifications for the formalistic approach are the law's certainty and predictability. However, the formalistic approach in traditional systems actually has quite a different origin. In the nonformalistic approach it is necessary to make a deduction from a major and a minor premise to obtain a normative conclusion. This procedure is carried out by human beings - commentators or judges. The traditional approach places less trust in human beings in general, and their individual sources in particular. It embodies the tacit assumption that it is better for the system's basic sources to carry out the deductive procedure themselves, and not leave it to the people who happen to be around at some particular time. Traditional systems therefore tend to use presumptions extensively, and this is what actually leads to their formalistic character.

As we concluded, the principles of a legal system are those of the cultural system in which it developed. Consequently, a radical non-formalistic approach cannot merely use the principles of the legal subsystem itself, but must attempt to go beyond them to the principles of the larger cultural system. Thus, although a Weberian separation between the legal subsystem and the cultural system does indeed reflect the formalist approach to legislation, it does not necessarily guarantee that the legal subsystem has no gaps. On the contrary, sometimes a legal system that tries to detach itself from the larger cultural system is less successful in coping with gaps, as it has abandoned a source that might have filled them in, while a non-formalist system might be more successful in that regard (as will be discussed further below). Actually, it is the formalist systems that have greater difficulty in removing their gaps. We may conclude, then, that the undetachable link between the values of the legal subsystem and those of the larger cultural system supports the non-formalist tendency to make use of the latter, at least in cases of normative gaps.

Part of the judicial process is the determination of the minor premise - the facts. The legal branch that determines how facts are decided in the legal process is evidence law. The laws of evidence determine which data should be considered "facts" and be presented in the legal proceedings, and which should not. From the viewpoint of Source Theory, the laws of evidence are a prototypical model of compartmentalization, as they are used to determine which of the existing data are admissible or inadmissible by the law, which is a source in the legal subsystem. Other sources are then applied to the admissible data - mainly the basic cognitive tools of the presiding judges.

The rejection of inadmissible data is a prototypical example of compartmentalization that is not based on a contradiction between data from different 
sources. The presentation of contradictory data, such as contrasting testimonies, is not prohibited. On the contrary, such data are often an important layer in the construction of the decision about the facts. The reasons for rejecting evidence are generally flaws in the way the evidence was gathered, such as "fruit of the poisonous tree" (in legal systems in which this rule prevails) or fact-based presumptions about the unreliability of the sources from which they were transmitted, as in the rule against hearsay.

Incidentally, in most legal systems, when a judge is faced with a question involving a law from a foreign legal system - especially if this law applies to the litigated case in virtue of the rules of private international law - the content of the law is considered a question of fact. It is decided according to the testimony of an expert witness, whose opinion is considered evidence of fact. Such witnesses are examined and cross-examined about their testimony in the same way as any other witnesses. This rule of evidence, although reasonable in and of itself, raises serious philosophical questions about the very distinction between law and fact. When a judge decides a case within her legal subsystem, isn't she determining a fact about the legal situation of this subsystem, just as the expert witness does in connection with a foreign legal system? It seems to me that this might actually be a correct description of the matter.

To return to the main issue, as soon as a judge is presented with an assortment of admissible data, she is faced with the problem that some of these data may be contradictory. Since all of them are admissible, they all belong to the system, and so her job is to decide among them. Most legal systems have few if any decision rules, so the decision must be made with the judge's discretion, which means her own sources. Since the legal subsystem does not provide any sources of this type, the judge's sources should be, once again, the basic sources of the cultural system to which she belongs, which the legal subsystem is also part of. This sort of decision, which is critical for the results of the proceedings, is thus based on the sources of the cultural system rather than any "autarchic" sources of the legal subsystem. Once the judge has come to a decision on the basis of these sources, the facts that were determined according to them are considered to have been proven.

At this stage we come to an issue that philosophers of law have dealt with more than any other - the application of the law to these proven facts. The legal-theoretic literature generally distinguishes between "easy cases" and "hard cases" in this regard. In terms of Source Theory, one could say that easy cases are those where the connection between the factual data and the legal data is established by the simple application of the power of judgment (Urteilskraft), using modus ponens - that is, when the facts clearly establish a special case of the antecedent of the general rule. In contrast, hard cases are those where there is no such match, and applying the rule to the facts requires interpretation, so that the judge or the commentator has to use additional sources and a more complex process of deduction. According to Hart (1961, Chapter 7), at this 
stage the judge is faced with an "open texture" where she has to use her judicial discretion. Since this discretion is free, whatever decision the judge makes is valid, and as soon as she makes the decision it becomes part of the law. According to Dworkin (1978, Chapter 2; 1985, Chapter 5), at this stage the judge must consider the principles of the legal system, which are an inseparable part of it, and try to find the proper balance among them. Dworkin claims that there is only one balance that is correct within the system, and if the judge fails to find it, her decision does not become part of the law.

We can reformulate this controversy in terms of Source Theory. Hart claims that judicial discretion is virtually free, but since "no man is an island", and even judges are part of the cultural system in which the law operates, judges normally make use of considerations that are derived from sources in this cultural system. Dworkin claims that judges must remain within the limits of the legal subsystem, but even he admits that its principles are those of "morality" (Dworkin 1978, Chapter 2) - that is, the principles are drawn from the cultural system (he uses the term "morality" because he is referring to WRS). Moreover, since, as we have seen, the legal subsystem's principles are almost always based on the values of the cultural system to which it belongs, there is practically no difference between them on the issue of the sources used by the judges. This lack of difference is extended to other areas by some of Hart's followers, who explicitly claim that even the judicial discretion have "sources" - such as the system's fundamental moral values, public opinion, and the judges' personal tendencies, all closely linked to the judge's cultural system - so that they are not absolutely free, as Hart seems to be claiming. In light of this analysis, the Hart-Dworkin controversy seems to be greatly diminished.

However, we still need to consider one aspect of this controversy that has remained open - whether every legal question has only one right answer (as the question is often formulated in the literature). Here, too, it seems that Source Theory can help us by greatly simplifying the discussion. Since we have concluded that judges must consider their cultural system's sources, we must distinguish between two levels of this system - the real one and the ideal one. We have shown above that the sources of the ideal system transmit data that are necessarily compatible (though not always accessible). Thus there cannot be two incompatible right answers to the same question within one ideal system. The real system, in contrast, is much less perfect. It embodies the aspiration to attain the purity of the ideal system, but the full achievement of this aspiration is utopian, and even its partial achievement is at best occasional. Therefore, Dworkin, who claims that there is one right answer to any legal question, is right on the ideal plane, but on the real plane it is not always possible to achieve one such answer, and so the law must recognize a range of possible answers as long as all of them are within the "zone of legitimacy" of the system (in the formulation of Barak 1989: 12). 
Dworkin himself seems to admit the ideal of nature of his "one right answer". Even if that answer remains unattainable, its existence is important to him as a tool for criticizing judicial decisions in "hard cases". In Hart's theory, where such cases create an "open texture", the judge seems to be free to decide, and any decision he makes will become the law, and will thus become "right". Thus, while in Dworkin's theory the existence of "one right answer" allows for criticism of the judge's decision, in Hart's theory its absence prevents any such criticism. This, indeed, is one of the arguments raised against Hart's theory. We can now support this argument by Source Theory. Only in a situation where there is a fixed system with agreed-upon basic sources can there be criticism or debate about judicial discretion (or anything else), since, as we have seen above, there can only be debates on the basis of common sources.

In many ways, the relation between law and morality in the normative sphere is quite similar to the relation between science and metaphysics in the descriptive sphere. On the ideal, utopian plane, in both disciplines, data should go from the basic to the derivative, or (to use Hegel's terms) from the abstract to the concrete. On the utopian plane, we know the basic moral principles, we know the correct formulae for how they should be balanced, and from those we safely derive the rules of law. Similarly, in the descriptive realm, we know the principles of reality, and they form the basis of any particular science that covers this or another part of reality. However, neither moral philosophers nor metaphysicians have reached the evident truths, and, given the society's urgent need for more everyday truths, the law has given up the quest for the solid rock for the norms and science has given up the quest for the solid rock for the facts. Neither lawyers nor scientists seek absolute truths, but rather something that will work. They have thus ignored the basic questions, taken some less basic premises for granted (usually without much awareness), and built their disciplines upon them. Indeed, in this sense both law and science are disciplines of despair.

To be sure, this despair has never occurred in reality, because in the development of human cultures, people did not even try to go from the abstract to the concrete but rather the other way around. The persons who established the law or the sciences took their underlying principles for granted, usually without even being aware of them. Only after concrete norms or facts were established did philosophers come and try to uncover the principles underlying them, whether in order to reaffirm them or in order to re-examine them. These lawyers and scientists often showed little interest in the discussions of those intellectuals who tried to explain the foundations of their daily work. However, the more their disciplines developed, the more they returned to the basic questions that they had originally sought to avoid. And so, from time to time they have had to deal with the principles that underlie their disciplines. They had to cope with philosophical questions, often without philosophers' professional tools 
We can now return to the discussion we started previously, where we defined "formalism" and "non-formalism" in legislation, and discuss the two concepts as they relate to judicial decisions and legal interpretation. Hart defined "formalism" according to his theoretical model, as a situation in which a judge does not recognize the existence of a "penumbra" and decides according to the existing rules, even if they do not really apply to the facts at hand. He defined the opposite approach, which he called "rule-skepticism", as a situation in which the judge does not recognize the rules and decides according to his own discretion, even when the rules fully apply to the facts at hand (Hart 1961, Chapter 7). In terms of Source Theory, Hart's model can be reformulated as follows: The formalist uses only the lower sources of the legal subsystem, while the rule-skeptic uses the basic sources of his own personal system and, to an even greater extent, those of his cultural system, which overlap with the higher sources of the legal subsystem (these seem to be the main sources of his discretion). This model is quite similar to our distinction between the formalist and non-formalist approaches to the nature of the legal subsystem itself, as discussed above. In the spirit of that discussion, we might say that formalism and non-formalism are not necessarily connected with the issue of the core versus the penumbra. Rather, we can now return, with greater support, to the model we suggested instead. Formalism is an approach that tends to use mainly the less general norms, while non-formalism tends to use the "higher" factual and normative layers they reflect, especially those of the legal subsystem itself, but also those of the cultural system as a whole. The distinction between the two approaches is not dichotomous, but rather ranges over a continuum.

In the epistemological realm, we argued that difference in the identity of the sources or the division of labor between them is not only a difference in thoughts but also a more fundamental difference in ways of thinking. Now we can similarly say that in the legal realm, the difference between the formalists and the non-formalists is not only in their substantive thoughts about the law and the facts, but is also a difference between two distinct legal ways of thinking (which Source Theory calls sources or source models). Indeed, it seems to me that this insight also agrees with the intuitions of experienced jurists.

We have so far examined the relations between two systems (Chapter Five) and the relations between a subsystem and a mother system (Chapter Six). We will now examine the functioning of another type of subsystem within the larger system, i.e. language (Chapter Seven). 
